University of Nebraska - Lincoln

DigitalCommons@University of Nebraska - Lincoln

Stephen Ducharme Publications

Research Papers in Physics and Astronomy

2004

\title{
Ferroelectricity at molecular level
}

L. M. Blinov

A. V. Bune

Peter A. Dowben

University of Nebraska-Lincoln, pdowben@unl.edu

Stephen Ducharme

University of Nebraska, sducharme1@unl.edu

Vladimir M. Fridkin

Institute of Crystallography, Russian Academy of Sciences, Moscow, fridkin@ns.crys.ras.ru

See next page for additional authors

Follow this and additional works at: https://digitalcommons.unl.edu/physicsducharme

Part of the Condensed Matter Physics Commons, and the Polymer and Organic Materials Commons

Blinov, L. M.; Bune, A. V.; Dowben, Peter A.; Ducharme, Stephen; Fridkin, Vladimir M.; Palto, S. P.; Verkhovskaya, K. A.; Vizdrik, G. V.; and Yudin, S. G., "Ferroelectricity at molecular level" (2004). Stephen Ducharme Publications. 60.

https://digitalcommons.unl.edu/physicsducharme/60

This Article is brought to you for free and open access by the Research Papers in Physics and Astronomy at DigitalCommons@University of Nebraska - Lincoln. It has been accepted for inclusion in Stephen Ducharme Publications by an authorized administrator of DigitalCommons@University of Nebraska - Lincoln. 


\section{Authors}

L. M. Blinov, A. V. Bune, Peter A. Dowben, Stephen Ducharme, Vladimir M. Fridkin, S. P. Palto, K. A. Verkhovskaya, G. V. Vizdrik, and S. G. Yudin 


\title{
FERROELECTRICITY AT MOLECULAR LEVEL
}

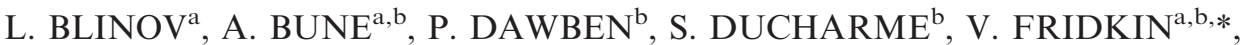 \\ S. PALTO ${ }^{\mathrm{a}}$, K. VERKHOVSKAYA ${ }^{\mathrm{a}}$, G. VIZDRIK $^{\mathrm{a}}$ and S. YUDIN ${ }^{\mathrm{a}}$ \\ a Shubnikov Institute of Crystallography, Russian Academy of Sciences, Leninskii pr. 59, \\ Moscow 119333, Russia; \\ ${ }^{\mathrm{b}}$ University of Nebraska-Lincoln, Lincoln, NE 68588-0111, USA
}

The synthesis of ultrathin ferroelectric nanostructures by the Langmuir-Blodgett method and their properties are reviewed. It is shown that ferroelectricity exists in one monolayer of the ferroelectric P(VDF-TrFE) copolymer, i.e., at the molecular level. The specific characteristics of switching of ultrathin ferroelectric films are established.

Keywords: Langmuir-Blodgett films; Ferroelectric polymer; Ultrathin ferroelectric films; Polarization switching

\section{INTRODUCTION}

In 1920, Valasek discovered that the crystals of Seignette (Rochelle) salt behave like a ferromagnet - they show the hysteresis of polarization and undergo phase transitions from the polar to the nonpolar phase (Valasek, 1921). In 1930s, Kurchatov suggested to call crystals similar to Seignette salt the "seignetoelectrics"; in the West, by analogy with ferromagnetics, these crystals are called ferroelectrics. Almost to until the end of the Second World War, the Seignette salt had remained the only representative of this new class of crystals possessing the particular polar direction. At the end of the Second World War, B.M. Vul and I.M. Goldman and, independently, Von Hippel et al. and Ogawa in Japan discovered a new ferroelectric - barium titanate. Several years later, Smolenskii et al. (Smolenskii et al., 1971) and Pepinsky and Mattias in the USA extended the list of ferroelectric crystals. Over the 80 years of the development of the field of ferroelectricity, hundreds of inorganic and organic crystals - ferroelectrics have been discovered. The ferroelectric properties were also revealed in liquid crystals (Pikin, 1981), semiconductors (Fridkin, 1976), thin films, ceramics (Scott, 1991; Tilley, 1996), and polymers (Yagi et al., 1980; Furukawa, 1984, 1989; Wang et al., 1988).

In 1940s, V.L. Ginzburg, proceeding from the Landau theory of phase transitions, developed the phenomenological theory of ferroelectricity. Somewhat later, similar results were also obtained by A. Devonshire. Later, various aspects of this theory

*Corresponding author. E-mail: fridkin@ns.crys.ras.ru 
were considered by E.M. Lifshitz, V.L. Indenbom, S.A. Pikin, A.P. Levanyuk, D.G. Sannikov et al. The phenomenological Landau-Ginzburg theory explained the main properties of ferroelectrics such as the nonlinear dependence of polarization on the applied field, dielectric hysteresis, the Curie-Weiss law (for second-order phase transitions), the behavior of spontaneous polarization, coercive field, and other parameters in the vicinity of the Curie and phase-transition temperatures, the critical (tricritical) points on the pressure-temperature diagram, and some other phenomena.

However, the theory failed to explain a group of other phenomena of these crystals. For example, the experimental strength of the coercive fields was lower by several orders of magnitude than the value predicted by this theory. The coercive field in thin ferroelectric films increased with a decrease of the film thickness (finite-size effect), but even in the thinnest films $(d \geq 600 \AA)$ obtained by magnetron sputtering and the sol-gel and other chemical methods, the coercive-field strength was considerably lower than the theoretically predicted value. With the discovery of domains in the Seignette salt by M.V. Klassen-Neklyudova, M.A. Chernysheva, and A.A. Shternberg and, especially, the study of domains and their role in the switching of polarization in barium titanate crystals performed by Merz (1954), it became possible to interpret this discrepancy. The point is that the Landau-Ginzburg theory does not take into account the role played by domains in the mechanism of ferroelectric switching. It turned out that it is the mechanism of domain nucleation and domain dynamics that plays the decisive role in the kinetics of switching. In order to switch a ferroelectric in accordance with the Landau-Ginzburg theory (conditionally called intrinsic switching) and to record experimentally the coercive-field strengths of the order of $10^{9} \mathrm{~V} \mathrm{~m}^{-1}$ following from this theory, one has to create the ferroelectric films so thin that the domain motion in these films would play only an insignificant role. At that time, the factors similar to the surface and depolarization energies seem to lead to the conclusion of the existence of a certain critical film thickness that contradicts the existence of ferroelectricity (Tilley, 1996). Until recently, no such critical thickness predicted by the theory has been observed experimentally.

Thus, the phenomenological Landau-Ginzburg theory fails to describe the switching mechanism of ferroelectric crystals and films associated with the domain dynamics (hereafter called the extrinsic mechanism). The Kolmogorov-Avrami-Ishibashi theory (Ishibashi, 1996) describes quite well the kinetics of extrinsic (domain) switching and explains its main experimental features, namely, the exponential dependence of the switching time $t_{0}$ on $E^{-1}$ (where $E$ is the external field) and a decrease in $t_{0}$ with temperature (at the phase-transition point, $t_{0}=0$ ).

\section{FERROELECTRIC POLYMERS}

Ferroelectric polymers have been known for more than 20 years. Films of the ferroelectric PVDF polymer and its $\mathrm{P}(\mathrm{VDF}-\mathrm{TrFE})$ copolymer were prepared from the solution by centrifuging (spinning). Usually, the thickness of the films obtained by spinning (hereafter referred to as spinning films) ranges within $1-10 \mu \mathrm{m}$, but some specific methods provide the synthesis of films as thin as $600 \AA$. Spinning films are complex composite material consisting of the amorphous phase with inclusions of polymer microcrystals. 
PVDF is a ferroelectric polymer consisting of carbon chains with $\mathrm{CH}_{2}-\mathrm{CF}_{2}$ groups spaced by $2.6 \AA$ (Fig. 1). The chains possess dipole moments normal to the chains and related to electronegative fluorine and electropositive hydrogen. In the $\beta$ phase, the chains form a quasihexagonal packing with spontaneous polarization $P \approx 0.13 \mathrm{C} \mathrm{m}^{-2}$. Switching and dielectric hysteresis are provided by the rotation of $\mathrm{H}-\mathrm{F}$ dipoles about carbon/hydrogen/chains.

The temperature of the phase transition from the ferro- to the paraelectric phase in PVDF is higher than the melting point. Therefore, the ferroelectric properties are studied mainly in $\mathrm{P}(\mathrm{VDF}-\mathrm{TrFE})$ copolymer, vinylidene fluoride, and trifluoroethylene with the structural formula $\left(\mathrm{CH}_{2}-\mathrm{CF}_{2}\right)_{n}-\left(\mathrm{CHF}-\mathrm{CF}_{2}\right)_{m}$. At a TrFE content lower than $50 \%$, this copolymer is a ferroelectric. Spontaneous polarization and the temperature of the phase transition from the ferro- to the paraelectric phase in this copolymer are lower than in PVDF, because the replacement of some hydrogen by fluorine reduces the dipole moment of the chain. Spinning polymer films show fast ferroelectric switching $(\sim 1 \mu \mathrm{s})$ dependent on the film thickness and electric-field strength.

In the best studied copolymers, the VDF:TrFE ratio is about 70:30. This copolymer is characterized by spontaneous polarization $P \approx 0.1 \mathrm{C} \mathrm{m}^{-2}$ and undergoes a first-order phase transition from the ferro- to the paraelectric phase at $T_{\mathrm{c}} \approx+100^{\circ} \mathrm{C}$ with a pronounced temperature hysteresis. The polymer structure changes in the phase transition. The trans-trans (TTTT) polar configuration (Fig. 1a) changes to the nonpolar transgauche (TGTG) one (Fig. 1b). The copolymer unit cell is somewhat larger than that of the PVDF polymer because the ionic radius of fluorine exceeds that of hydrogen.

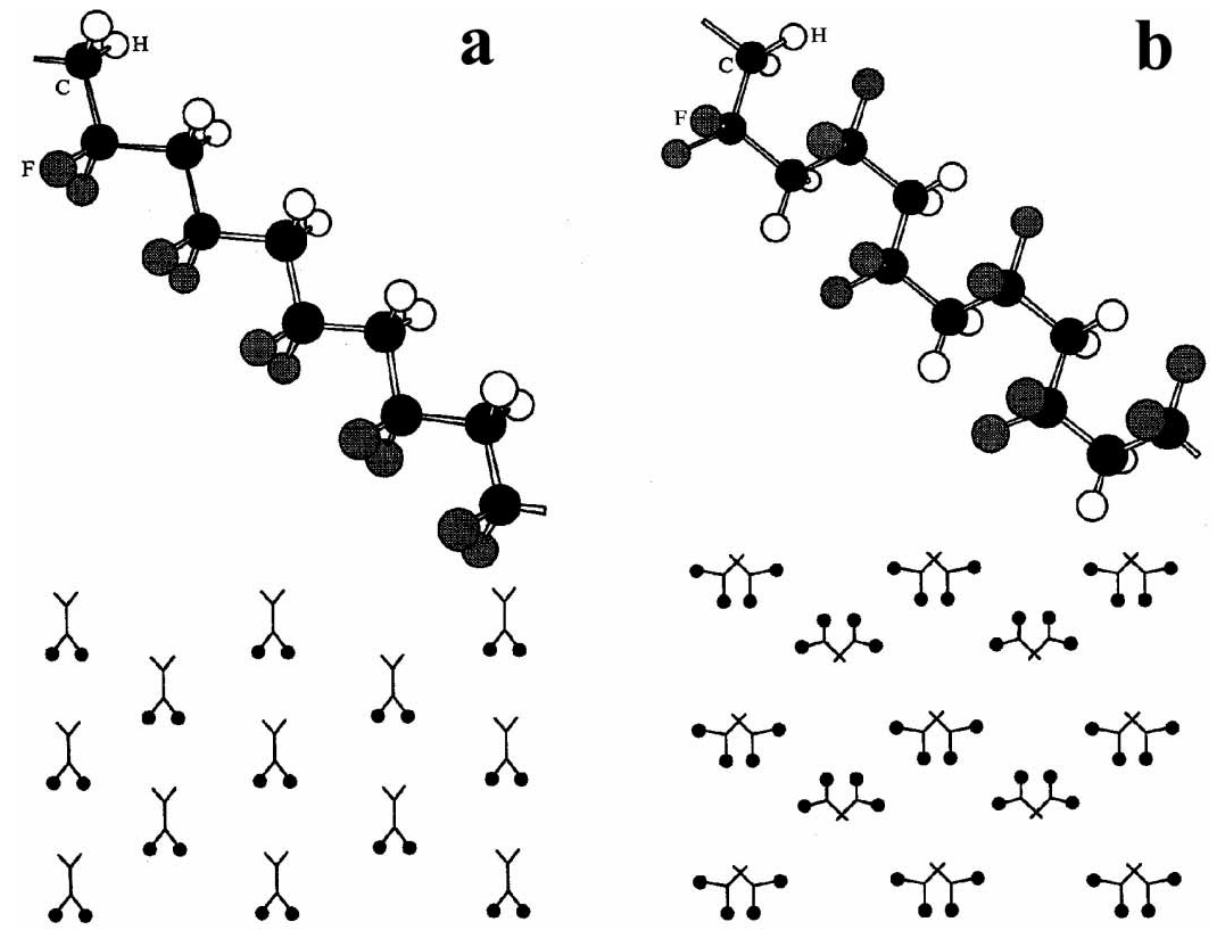

FIGURE 1 (a) PVDF structure in the ferroelectric phase (trans-trans conformation). (b) PVDF structure in the paraelectric phase (trans-gauche conformation). 
In spinning films of the $\mathrm{P}(\mathrm{VDF}-\mathrm{TrFE})$ (75:25) copolymer, the finite-size effect is observed. For films with the thickness $L>600 \AA$, an increase in the coercive field for thinner films was observed. Since it is impossible to synthesize spinning films thinner than $600 \AA$, the finite-size effect for thinner copolymer films was not studied.

In 1995, crystalline films of ferroelectric P(VDF-TrFE) copolymers were grown by the Langmuir-Blodgett method. These films possess ferroelectric properties close to those of bulky films, including switching and a first-order ferroelectric phase transition (Bune et al., 1995; Palto et al., 1995). The use of the Langmuir-Blodgett method allowed one to obtain, for the first time, ultrathin ferroelectric films with a thickness of one monolayer $(5 \AA)$. This opened the possibility for studying the finite-size effect at the molecular level and checking the existence of the critical film thickness.

Langmuir-Blodgett (LB) films are the well-known structures obtained by the successive transfer of monolayers from the gas-liquid interface onto a solid substrate. One of the most important characteristics of this method is the simultaneous control of the LB-film structure with an accuracy of one monolayer and of the molecules and their orientations in a monolayer.

High-quality thin films of the P(VDF-TrFE) $(70: 30)$ ferroelectric are obtained by the horizontal Langmuir-Schaeffer method (modification of the LB method) schematically illustrated in Fig. 2a under the precision control of a nanostructure. Ferroelectric LB films are obtained by dissolving the copolymer in a $0.01 \%$ aqueous solution of dimethyl sulfoxide. The solution in thrice distilled water was studied by constructing the pressure-area isotherm (Fig. 2b), which provided the determination of the necessary conditions for obtaining a molecular close packing in a monolayer on the solution surface. At room temperature, these conditions were met at the surface pressure $5 \mathrm{~m} \mathrm{~N} \mathrm{~m}^{-1}$ and the area $5.7 \AA^{2}$ per structural unit. (The films prepared at considerably higher pressures were inhomogeneous and consisted of more than one monolayer.) To perform electric measurements, the monolayers were transferred onto glass or silicon substrates coated with aluminum. The role of the second electrode was played by aluminum deposited onto the film surface. The films transferred onto the graphite substrate obtained by cleaving graphite crystals were studied in a scanning tunneling microscope (STM). The X-ray, neutron, and electron diffraction studies were performed on LB films deposited onto differently oriented single-crystal substrates.

The structure of ferroelectric LB films was studied in an STM at atomic resolution (Palto et al., 1996; Bune et al., 1998; Choi et al., 1998) by the methods of X-ray, neutron, and electron diffraction (Choi et al., 1998, 1999; Borca et al., 1999) and also in a scanning electron microscope. The films show high crystallinity and are obviously oriented. Spontaneous polarization is normal to the film surface. Nevertheless, the existence of differently oriented domains with different orientations of parallel chains cannot be excluded. Ferroelectric LB films undergo a first-order ferroelectric phase transition. The character of this phase transition is confirmed by the existence of the temperature hysteresis of the dielectric constant, complete switching of polarization below $T_{\mathrm{c}}=80^{\circ} \mathrm{C}$, and double hysteresis loops in the temperature range between $T_{0}$ and the critical point at $145^{\circ} \mathrm{C}$ (Ducharme et al., 1998).

STM studies at the atomic resolution show the perfect structure of the films with the carbon chains parallel to the film plane (Fig. 3). The monomers are spaced by about $2.6 \AA$, indicating the high order along the chains and good accord with the structural data obtained earlier for bulky films. 

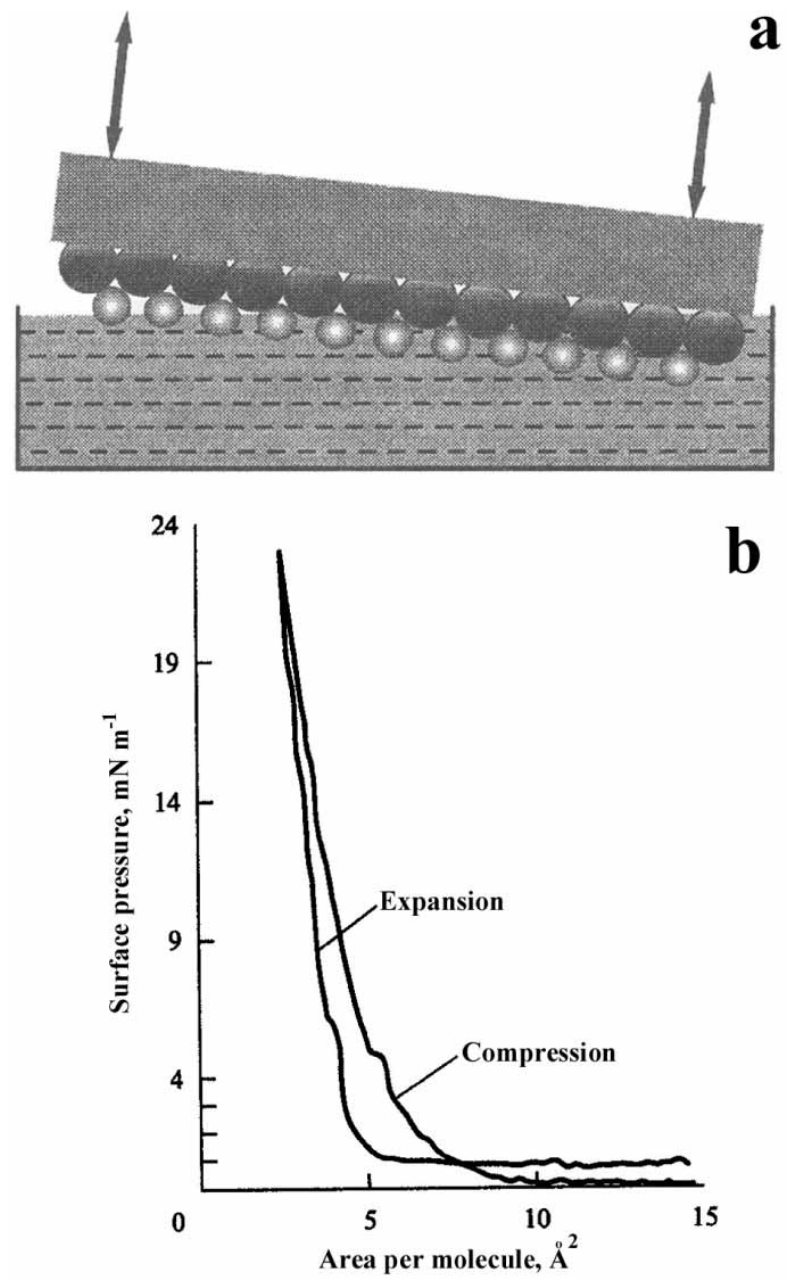

FIGURE 2 (a) Schematic illustration of the Langmuir-Schaeffer method and (b) the pressure-area isotherm for $\mathrm{P}(\mathrm{VDF}-\mathrm{TrFE})(70: 30)$.

Both X-ray (Choi et al., 1999) and neutron (Borca et al., 1999) diffraction show high ordering along the [010] direction (normal to the film surface) with the interlayer spacings (unit-cell period $b$ ) equal to $4.5 \AA$, which is consistent with the structure of spinning films. Figure 4 shows hysteresis in the temperature dependence of the period $b$ for 5 (Fig. 4a) and 150 (Fig. 5b) monolayers. This temperature hysteresis also observed in the spinning films is the consequence of the metastable coexistence of the ferro- and paraelectric phases in the first-order phase transition.

Ferro-, piezo-, and pyroelectric properties of LB films are close to the corresponding properties of spinning films (see Table I).

\section{TWO-DIMENSIONAL FERROELECTRIC FILMS}

Thus, the Langmuir-Blodgett method is the technology of growing ferroelectric crystalline films, including ultrathin ones. Figure 5 illustrates switching in P(VDF-TrFE) 

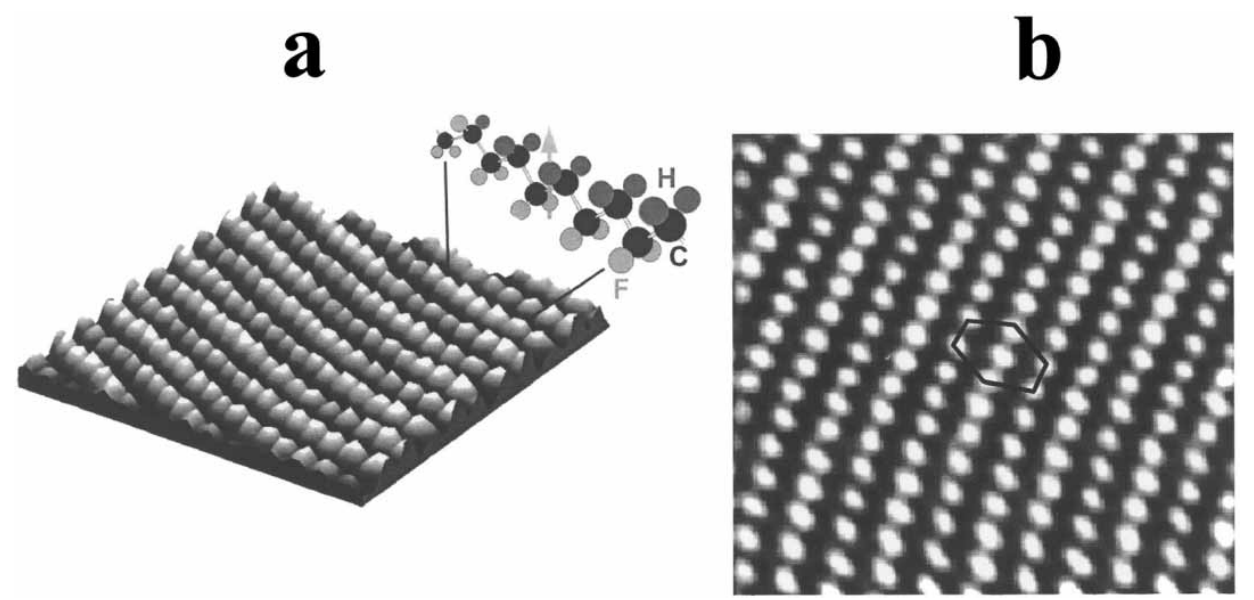

FIGURE 3 (a) P(VDF-TrFE) monolayer on a graphite substrate in an STM and (b) quasihexagonal structure of a monolayer in an STM.

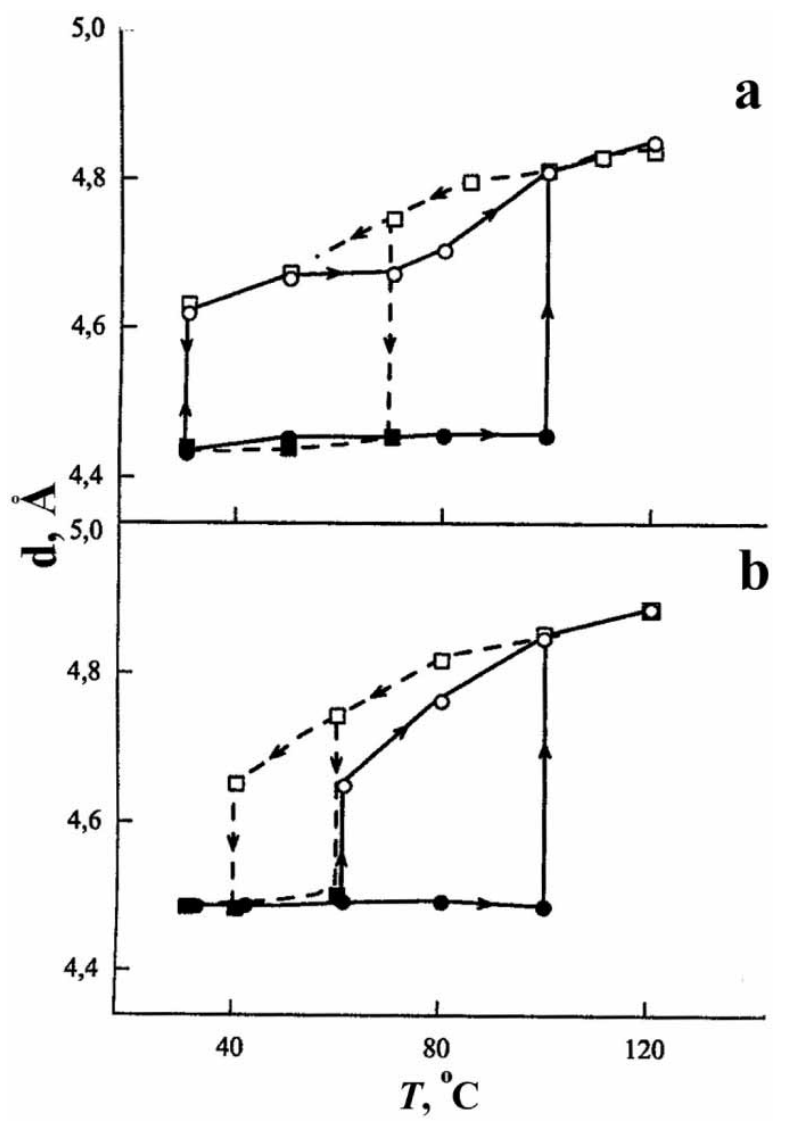

FIGURE 4 Variation of the lattice parameter along the [010] direction in the vicinity of the phase transition for Langmuir-Blodgett P(VDF-TrFE) films consisting of: (a) 5 monolayers and (b) 150 monolayers. 


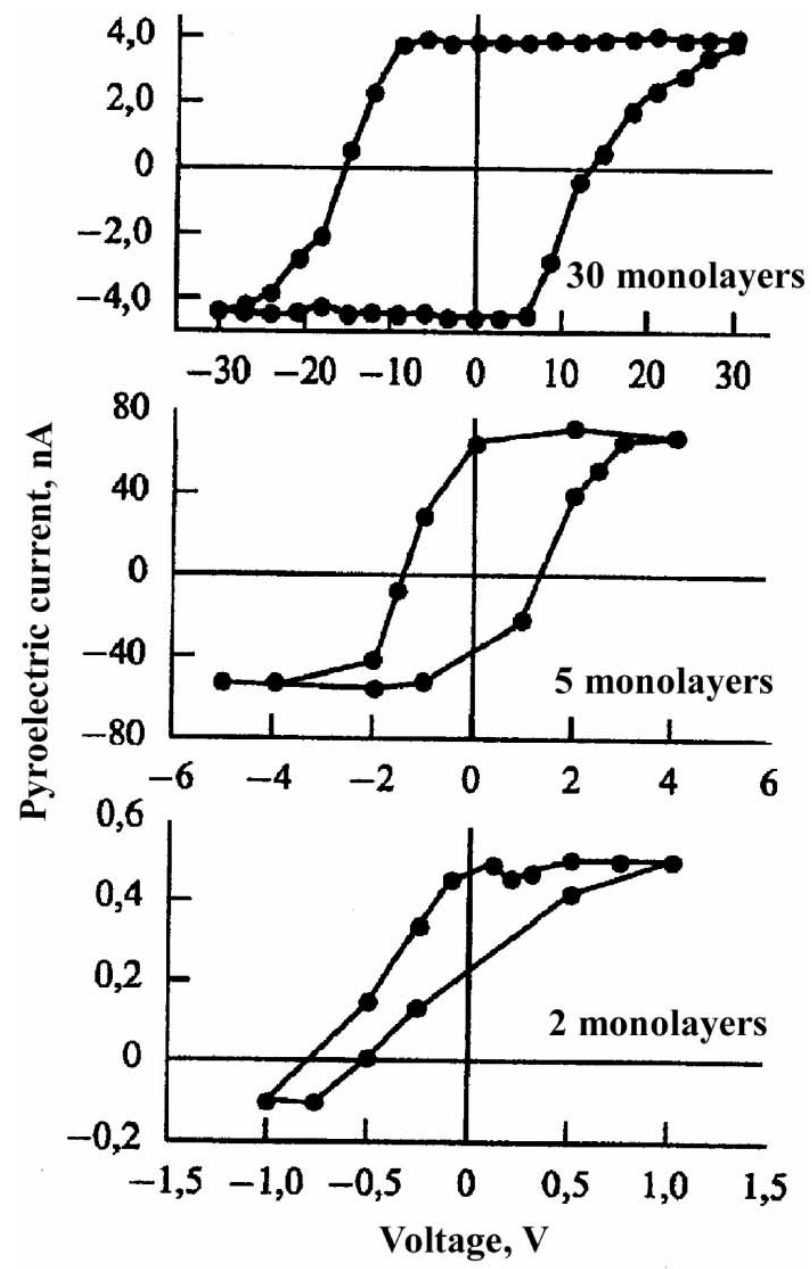

FIGURE 5 Polarization hysteresis in LB films (2, 5, and 30 monolayers).

TABLE I Piezoelectric and pyroelectric coefficients of Langmuir-Blodgett and spinning films

\begin{tabular}{lccc}
\hline Material & $d_{33}, 10^{-9} \mathrm{~m} \mathrm{~V}^{-1}$ & $p_{3}, \mu \mathrm{Cm}^{-2} \mathrm{~K}^{-1}$ & $P_{\mathrm{s}, \mathrm{Cm}^{-2}}$ \\
LB films & $20 \pm 2$ & $20 \pm 4$ & 0.1 \\
Spinning films & 40 & 35 & 0.1 \\
\hline
\end{tabular}

( $70: 30)$ films with the thickness $150 \AA$ ( 30 monolayers), $25 \AA$ (5 monolayers), and $10 \AA$ ( 2 monolayers). The corresponding hysteresis loops were obtained by the Chynoweth method. Similar hysteresis loops were also observed by the Sawyer-Tower method. The hysteresis loop for two monolayers is obviously nonsymmetric (because of the influence of the substrate) and shows polarization switching. In the tunneling microscope, it was possible to observe the effect of the switching field on the behavior of a monolayer on a graphite substrate. Figure 6 illustrates the above stated at a switching voltage 


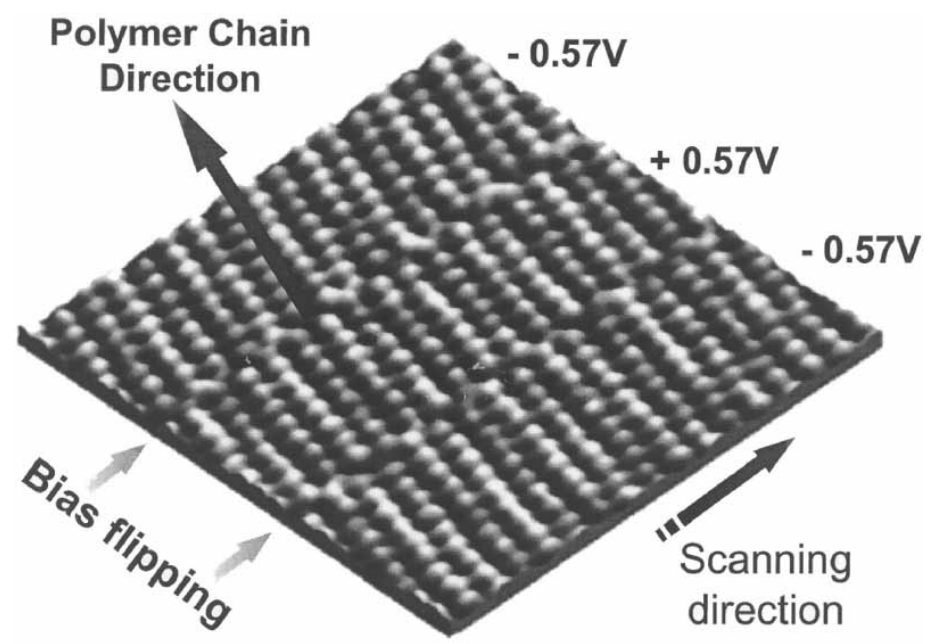

FIGURE 6 Effect of the switching field on one P(VDF-TrFE) monolayer (in a scanning tunneling microscope).

of $0.57 \mathrm{~V}$ (the corresponding field is $E \approx 1.5 \times 10^{9} \mathrm{~V} \mathrm{~m}^{-1}$ ). ${ }^{1}$ These results lead to two important conclusions. First, no critical thickness predicted for ferroelectric polymers was observed (Tilley, 1996). Ferroelectric polarization and its switching are observed in one monolayer. Second, the coercive field $E_{\mathrm{c}}$ in an ultrathin layer attains a value close to that theoretically predicted by the Landau-Ginzburg theory. In this case, $E_{\mathrm{c}}$ is constant in the thickness range from 30 to 2 monolayers (Fig. 7). In this range, a finite-size effect is not observed either for $E_{\mathrm{c}}$ or other parameters (Curie temperature, spontaneous polarization, etc.). In this case, the effect of the substrate is not important for a film with the thickness exceeding five monolayers, because the hysteresis loops turn out to be symmetric. An ultrathin film behaves as a two-dimensional ferroelectric whose existence was predicted by Onsager and Landau (Landau and Lifshitz, 1964). On this basis, it was concluded (Blinov et al., 2000; Ducharme et al., 2000) that switching in two-dimensional ferroelectric films is "intrinsic," in other words, domains play no essential part in switching.

This assumption was verified by studying the switching kinetics of ultrathin twodimensional P(VDF-TrFE) (70:30) films. The switching kinetics was studied by the Merz method and by the direct measurements of polarization as a function of the time of the external-field application.

Thick LB films (100 or more monolayers) show the kinetics described in the literature and characterized by the exponential dependence of the reciprocal switching time on the switching field

$$
t_{0}^{-1}=t_{0 m}^{-1} \exp \left(E / E_{0}\right)
$$

where $t_{0 m}$ is the constant, and a decrease in $t_{0}$ with the temperature is well consistent with the Kolmogorov-Avrami-Ishibashi theory. Consequently, the coercive field $E_{\mathrm{c}}$

\footnotetext{
${ }^{1}$ J. Zhang, Department of Physics, Florida International University, USA, private communication.
} 


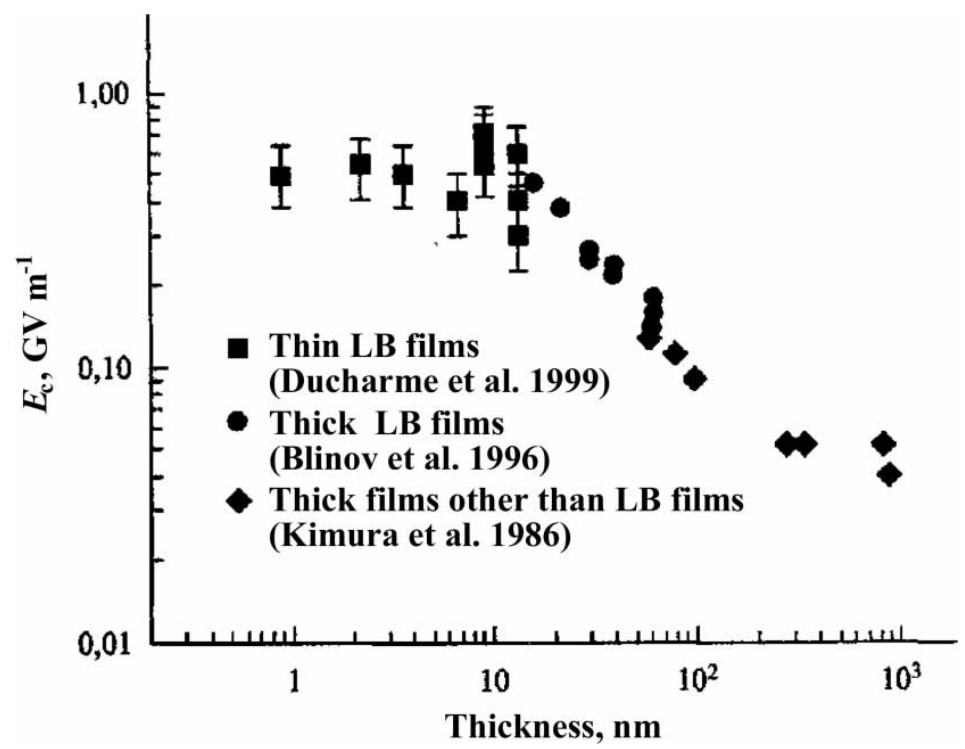

FIGURE 7 Coercive field versus thickness of LB and spinning films of a ferroelectric polymer.

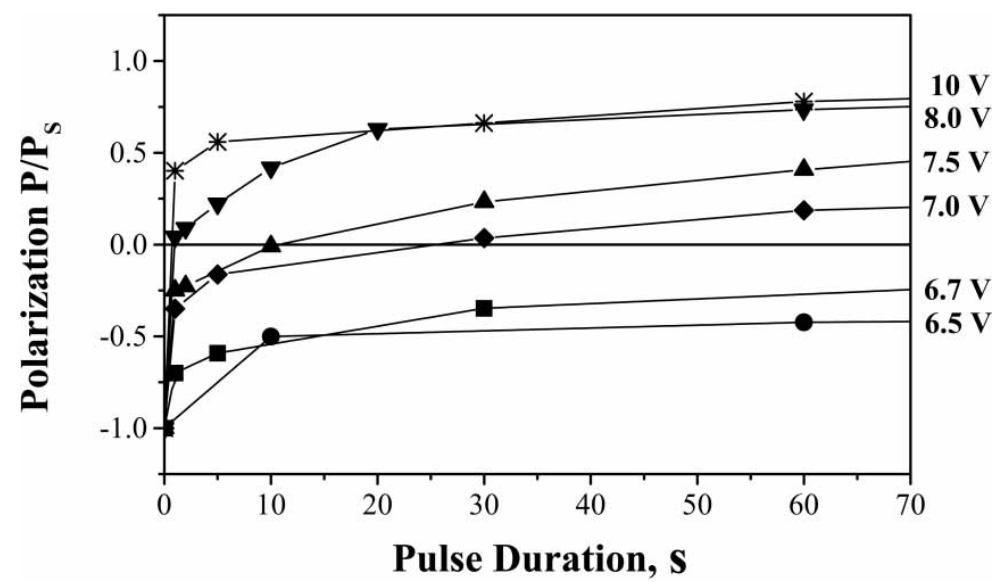

FIGURE 8 Polarization $P / P_{\mathrm{s}}(P$ is spontaneous polarization at $t=0)$ as a function of time at different voltages. Film consisting of 10 monolayers. Coercive voltage $V_{\mathrm{c}}=6.7 \mathrm{~V}$ (coercive field $E_{\mathrm{c}}=1.3 \times 10^{9} \mathrm{~V} \mathrm{~m}^{-1}$ ).

of these films is much lower than the value predicted by the Landau-Ginzburg theory and shows the finite-size effect (see Fig. 7).

On the contrary, ultrathin two-dimensional films (not thicker than 30 monolayers) show instead of the kinetics described by Eq. (1) an unusual kinetics never observed in ferroelectrics earlier. This kinetics is characterized by two features. Switching takes place only in the fields $E>E_{\mathrm{c}}$. At $E<E_{\mathrm{c}}$, no switching is observed; at $E \rightarrow E_{\mathrm{c}}$, switching is of the critical nature and the switching time $t_{0}$ dramatically increases.

Figure 8 shows the switching curves $P / P_{\mathrm{s}}=P / P_{\mathrm{s}}(t)$ for a film consisting of 10 monolayers with the coercive voltage $V_{\mathrm{c}}=6.7 \mathrm{~V}$ (and the coercive field $E_{\mathrm{c}} \approx 1.3 \times 10^{9} \mathrm{~V} \mathrm{~m}^{-1}$ ). It is seen that switching takes place only at voltages $V>6.7 \mathrm{~V}$. Figure 9a shows the dependence of $t_{0}^{-1}$ on the switching field $E / E_{\mathrm{c}}$ for films consisting of 10,30 , and 100 
monolayers. The critical nature of the behavior of $t_{0}$ at $E=E_{\mathrm{c}}$ is seen for films consisting of 10 and 30 monolayers. Film consisting of 100 monolayers shows the conventional exponential dependence $t_{0}^{-1}=t_{0}^{-1}(E)(1)$. No critical behavior at $E / E_{\mathrm{c}}=1$ is observed in this case. Figure $9 \mathrm{~b}$ shows an increase in $t_{0}$ with temperature for the films consisting of 10 and 30 monolayers. This behavior is opposite to the behavior following from the mechanism of extrinsic (domain) switching. On the contrary, film consisting of 100 monolayers shows a slow decrease in $t_{0}$ with temperature.
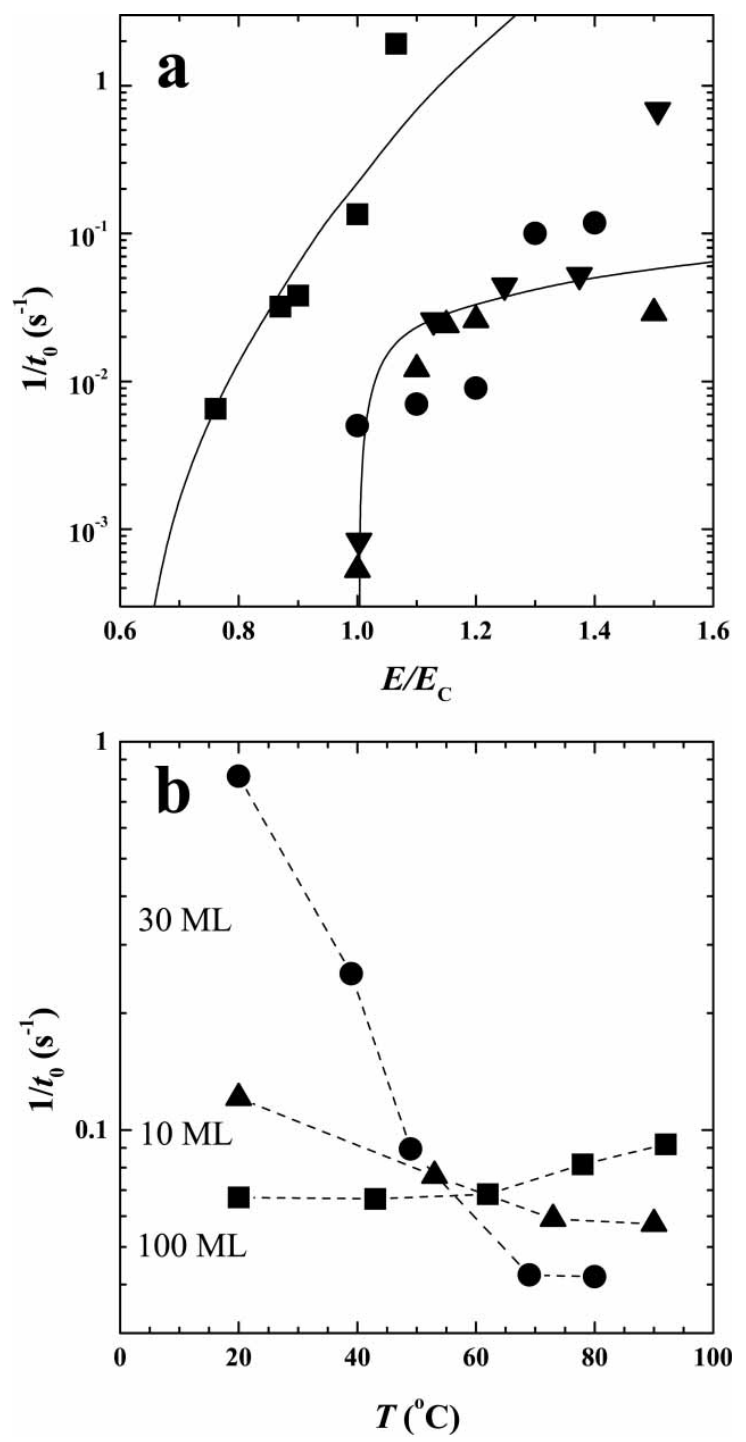

FIGURE 9 (a) Reciprocal switching time $t_{0}^{-1}$ versus field $E / E_{\mathrm{c}}\left(E_{\mathrm{c}}\right.$ is the coercive field) for films consisting of 10 monolayers (triangles), 30 monolayers (circles), and 100 monolayers (squares). The theoretical curves are constructed for $\xi=5 \times 10^{11} \mathrm{Vm} \mathrm{s} \mathrm{C}^{-1}, \beta=-1.1 \times 10^{12} \mathrm{~m}^{5} \mathrm{C}^{-2} \mathrm{~F}^{-1}, \gamma=6 \times 10^{13} \mathrm{~m}^{9} \mathrm{C}^{-4} \mathrm{~F}^{-1}$ (Blinov et al., 2000) and (b) reciprocal switching time $t_{0}^{-1}$ versus temperature. 


\section{KINETICS OF FERROELECTRIC SWITCHING ACCORDING TO LANDAU AND KHALATNIKOV}

Thus, the study of the switching kinetics of ultrathin two-dimensional ferroelectric $\mathrm{P}(\mathrm{VDF}-\mathrm{TrFE})$ films shows that switching in these films cannot be described by the extrinsic Kolmogorov-Avrami-Ishibashi mechanism and requires another interpretation.

Vizdrik et al. made an attempt to interpret this kinetics based on the Lagrange equation more often referred to as the Landau-Khalatnikov equation. Earlier, the switching kinetics was considered based on the Landau-Khalatnikov equations for ferroelectrics that undergo a second-order phase transition (Ricinschi et al., 1998). The solution of the Landau-Khalatnikov equation was obtained for switching of ferroelectrics undergoing a first-order phase transition. In this case, the LandauKhalatnikov equation has the form

$$
\xi \frac{\mathrm{d} P}{\mathrm{~d} t}=-\frac{\partial F}{\partial P}=-\alpha P-\beta P^{3}-\gamma P^{5}+E
$$

Here $P$ is spontaneous polarization, $F$ is the free energy, $\alpha, \beta$, and $\gamma$ are the well-known Landau-Ginzburg coefficients, $\xi$ is the phenomenological parameter (damping constant) determining the switching rate. The Landau-Khalatnikov equation in form (2) describes the kinetics of intrinsic switching not related to domain motion. The solution of Eq. (2) shows the critical nature of ferroelectric switching at $E=E_{\mathrm{c}}$ and $T=T_{1}\left(T_{1}\right.$ is the temperature of the first-order phase transition):

$$
t_{0}^{-1}=\frac{\beta^{2}}{6.3 \gamma \xi}\left(\frac{E}{E_{\mathrm{c}}}-1\right)^{1 / 2}\left(1-\frac{T-T_{0}}{T_{1}-T_{0}}\right)^{1 / 2} .
$$

It follows from Eq. (3) that the switching time $t_{0}$ tends to infinity at $E \rightarrow E_{\mathrm{c}}$ and $T \rightarrow T_{1} \quad\left(T_{0}\right.$ is the Curie-Weiss temperature). The analysis of the LandauKhalatnikov equation shows that switching can take place only at $E>E_{\mathrm{c}}$. The solid lines in Fig. 9a show the theoretical dependence of $t_{0}^{-1}$ on $E / E_{\mathrm{c}}$ for LB films consisting of 10,20 , and 100 monolayers. For the films consisting of 10 and 30 monolayers, the theoretical curve was constructed using Eq. (3), for the film consisting of 100 monolayers, with the aid of Eq. (1). It is seen that the thick film at $E=E_{\mathrm{c}}$ shows no specific features. Thus, the Landau-Khalatnikov equation explains quite well two main characteristics of the switching kinetics of ultrathin two-dimensional films first, the critical nature of switching at $E=E_{\mathrm{c}}$, and $T=T_{1}$ and, second, the absence of switching at $E<E_{\mathrm{c}}$. This is an additional confirmation of the intrinsic (domainfree) nature of switching in domain films. It is seen from Fig. 9 that in the vicinity of $E=E_{\mathrm{c}}$, slow switching of an ultrathin film takes place (in accordance with the Landau-Khalatnikov equation, $t_{0} \rightarrow \infty$ at $E \rightarrow E_{\mathrm{c}}$ ). Even if $E \gg E_{\mathrm{c}}$, the switching time for ultrathin films is $t_{0} \geq 10^{-3} \mathrm{~s}$, i.e., is longer by several orders of magnitude that for spinning films or thick LB films demonstrating the conventional exponential dependence $t_{0}^{-1}=t_{0}^{-1}(E)$ in Eq. (1). It is seen from Eq. (3) that the intrinsic-switching time within the phenomenological Landau-Khalatnikov approach depends on the kinetic coefficient $c$. The latter depends on the mechanism of the domain-free switching 
that cannot be interpreted within the Landau-Khalatnikov approach. Thus, for a uniaxial ferroelectric that undergoes an order-disorder phase transition, we have

$$
\xi=\frac{2 V}{e p_{0}}(2 \pi m k T)^{1 / 2} \exp \left(\frac{2 U}{k T}\right)
$$

where $p_{0}$ is the dipole moment of the unit cell, $V$ is the unit-cell volume, $m$ is the ion mass, and $U$ is the barrier energy. Substituting $p_{0}=7 \times 10^{-30} \mathrm{Cm}, m=1.7 \times$ $10^{-27} \mathrm{~kg}, \quad \mathrm{~V} \approx 100 \AA^{3}$, and $U=0.46 \mathrm{eV}$ into Eq. (4) for PVDF, we obtain $\xi \approx 10^{11} \mathrm{~V} \mathrm{~m} \mathrm{~s} \mathrm{C}^{-1}$. This $\xi$ value, and, correspondingly, also the $t_{0}$ value, are in good accord with the experimental data for ultrathin LB films (Fig. 9a). A similar $\xi$ value is also obtained for PVDF films from the Fokker-Planck equation. ${ }^{2}$

\section{CONCLUSION}

The discovery and study of ultrathin two-dimensional ferroelectric films of P(VDFTrFE) copolymer gave rise to two most important results. First, the absence of the critical film thickness predicted earlier is proven. Ferroelectricity exists in one monolayer, i.e., at the molecular level. Second, it is shown that the kinetics of ferroelectric switching of ultrathin films is of the critical nature and well described by the Landau-Khalatnikov equation. This signifies that switching is of the intrinsic nature not associated with domain motion. The study of nanostructures of ferroelectric films is only at the very beginning. There is no doubt that the Langmuir-Blodgett and other methods would provide the synthesis of nanostructures of other ferroelectric materials. Thus, recently (Tybell et al., 1999), perovskite-like PZT films with a thickness of $40 \AA$ (10 unit-cell parameters) were obtained by magnetron sputtering. The study of ferroelectric films at the molecular level opens new vistas for both fundamental and applied science.

\section{References}

Blinov, L.M., Fridkin, V.M., Palto, S.P., Bune, A.V., et al. (2000). Two-dimensional ferroelectrics. Physics Uspekhi, 43, 243.

Borca, C.N., Choi, J., Adenwalla, S., Ducharme, S., et al. (1999). Influence of dynamical scattering in crystalline poly(vinylidene fluoride-trifluoroethylene) copolymers. Appl. Phys. Lett., 74, 329.

Bune, A., Ducharme, S., Fridkin, V., Palto, L.B.S., et al. (1995). Novel switching phenomena in ferroelectric Langmuir-Blodgett films. Appl. Phys. Lett., 67, 3835.

Bune, A.V., Fridkin, V.M., Ducharme, S., Blinov, L.M., et al. (1998). Two-dimensional ferroelectric films. Nature, 391, 874.

Choi, J., Dowben, P.A., Ducharme, S., Fridkin, V.M., et al. (1998). Lattice and electronic band structure changes across the surface ferroelectric transition. Phys. Lett. A, 249, 505.

Choi, J., Dowben, P.A., Borca, C.N., Adenwalla, S., et al. (1999). Evidence of dynamic Jahn-Teller distortions in two-dimensional crystalline molecular films. Phys. Rev. B, 59, 1819.

Ducharme, S., Bune, A.V., Blinov, L.M., Fridkin, V.M., et al. (1998). Critical point in ferroelectric LangmuirBlodgett polymer films. Phys. Rev. B, 57, 25.

Ducharme, S., Fridkin, V.M., Bune, A.V., Palto, S.P., et al. (2000). Intrinsic ferroelectric coercive field. Phys. Rev. Lett., 84, 175.

\footnotetext{
${ }^{2} \mathrm{~V}$. Stephanovich, University of Opole, Poland, private communication.
} 
Fridkin, V.M. (1976). Segnetoelektriki-Poluprovodniki (Russian). Nauka, Moscow.

Furukawa, T. (1984a). Phenomenological aspect of a ferroelectric vinylidene fluoride/trifluoroethylene copolymer. Ferroelectrics, 57, 63.

Furukawa, T. (1984b). Phenomenological aspect of a ferroelectric vinylidene fluoride/trifluoroethylene copolymer. Ferroelectrics, 57, 63.

Furukawa, T. (1989). Ferroelectric properties of vinylidene fluoride copolymers. Phase Transitions, 18, 143.

Ishibashi, Y. (1996). Polarization reversals in ferroelectrics. In: Paz de Araujo, C., Scott, J.F. and Taylor, G.F. (Eds.), Ferroelectric Thin Films: Synthesis and Basic Properties, Volume 10, Chapter 5, 135. Gordon and Breach, Amsterdam.

Landau, L.D. and Lifshitz, E.M. (1964). Statisticheskaya Fizika (Russian), Volume 5. Nauka, Moscow.

Merz, W.J. (1954). Domain formation and domain wall motions in ferroelectric $\mathrm{BaTiO}_{3}$ single crystals. Phase Transitions, 95, 690.

Palto, S., Blinov, L., Bune, A., Dubovik, E., et al. (1995). Ferroelectric Langmuir-Blodgett films. Ferroelectric Lett., 19, 65.

Palto, S., Blinov, L., Dubovik, E., Fridkin, V., et al. (1996). Ferroelectric Langmuir-Blodgett films showing bistable switching. Europhys. Lett., 34, 465.

Pikin, S.A. (1981). Strukturnye Prevrascheniya v Zhidkih Kristallah (Russian). Nauka, Moscow.

Ricinschi, D., Harnagea, C., Papusoi, C., Mitoseriu, L., et al. (1998). Analysis of ferroelectric switching infinite media as a Landau-type phase transition. J. Phys.: Condens. Matter, 10, 477.

Scott, J.F. (1991). Phase transitions in ferroelectric thin films. Phase Transitions, 30, 107.

Smolenskii, G.A., Isupov, V.A.B.B.A., Krainik, N.N., et al. (1971). Ferroelectrics and Antiferroelectrics. Nauka, St. Petersburg.

Tilley, D.R. (1996). Finite-size effect on phase transitions in ferroelectric films. In: de Araujo, C.P., Scott, J.F. and Taylor, G.F. (Eds.), Ferroelectric Thin Films: Synthesis and Basic Properties, 11. Gordon and Breach, Amsterdam.

Tybell, T., Ahn, C.H. and Triscone, J.-M. (1999). Ferroelectricity in thin perovskite films. Appl. Phys. Lett., $75,856$.

Valasek, J. (1921). Piezo-electric and allied phenomena in rochelle salt. Phys. Rev., 17, 475.

Wang, V.M., Helbert, J.M. and Glass, A.M. (1988). The Application of Ferro-electric Polymers. Chapman and Hall, New York.

Yagi, T., Tatemoto, M. and Sako, J. (1980). Transition behavior and dielectric properties in trifluoroethylene and vinylidene fluoride copolymers. Polymer J., 12, 209. 
Copyright of Phase Transitions is the property of Taylor \& Francis Ltd and its content may not be copied or emailed to multiple sites or posted to a listserv without the copyright holder's express written permission. However, users may print, download, or email articles for individual use. 